\title{
ANALISI RADIOATTIVA DELLE ROCCE COL METODO FOTOGRAFICO (*)
}

\author{
G. Imbò - L. Casertano
}

I. - In due note precedenti ( $\left.{ }^{1}\right)$ è stato indicato il metodo per calcolare, dalle distribuzioni statistiche delle particelle $"$, raccolte su lastre fotografiche, in funzione della proiezione sulla lastra del percorso della particella nell'emulsione, non solo il rapporto in peso delle concentrazioni di Uranio $\left(C_{\mathrm{U}}\right)$ rispetto a quelle del Torio $\left(C_{\mathrm{Th}}\right)$, ma anche il contenuto in Uranio e Torio della roccia in esame: presupponendo però raggiunte le condizioni di equilibrio radioattivo in ognuna delle famiglie.

Ora nell'applicare il metoro a lave vesuviane si ̀̀ voluto prescindere da qualsiasi ammissione, e si è arrivato a riconoscere - almeno in linea teorica - la possibilità di verificare addirittura le condizioni in cui si trovano vari elementi delle famiglie radioattive rispetto a quelle che dovrebbero essere le condizioni di equilibrio.

La formula [5] della seconda delle note citate

$$
n=\frac{N}{4} \mu_{1} R\left(1-\frac{r}{\mu_{2} R}\right)^{2}
$$

consente di ricavare, per spessori della sostanza equivalenti a percorsi nell'aria maggiori di quello $(R)$ dell'elemento che si considera, il numero $(N)$ di particelle $\alpha$ emesse per $\mathrm{cm}^{3}$ di sostanza in base al numero (n) di proiezioni, contate per $\mathrm{cm}^{2}$, maggiori di $r$, essendo $: i_{1}$ il coefficiente di frenamento della sostanza radioattiva, e u. quello dell'emulsione.

La [1], come è stata estesa alle famiglie radioattive in equilibrio, può genericamente estendersi a elementi qualsiasi radioattivi, della stessa famiglia o meno, e, nel primo caso, non necessariamente in equilibrio. Nel caso generale evidentemente anche per $N$ devono assumersi valori che possono anche non essere uguali per i singoli elementi. La formula, quindi, diventa:

$$
n=\frac{\mu_{1}}{4} \sum_{\mathrm{i}} N_{\mathrm{i}} R_{\mathrm{i}}\left(1-\frac{r}{\mu_{\mathrm{2}} R_{\mathrm{i}}}\right)^{2}
$$

(*) Comunicazione presentata al Convegno dell ${ }^{*}$ ssociazione Geofisica Italiana, tenuto a Roma il 17.18 giugno 1953. 
con la sommatoria estesa a tutti gli elementi il cui percorso nell'aria $\left(R_{i}\right)$ è maggiore dell'equivalente in aria $\left(r / \mu_{2}\right)$ della proiezione $(r)$.

2. - Con le tracce contate su una lastra $\dot{e}$ possibile ricavare dalla [2] tante equazioni quante sono le proiezioni $r$ considerate: le incognite risultano le $N_{\mathrm{i}}$. Risulta chiaro che $\dot{e}$ conveniente cercare di ridurre il numero delle incognite, considerando proiezioni che sono minori di determinati valori dei percorsi delle particelle $\alpha$. D'altra parte la differenza fra le proiezioni deve essere tale da consentire facilmente il conteggio delle tracee, con la minore possibilità di confusione: il che porta alla diminuzione del numero delle equazioni.

Si ritiene conveniente il conteggio dei numeri $n_{1}, n_{9}, \ldots \ldots, n_{10}$ di tracce aventi proiezioni maggiori rispettivamente di $0,60 \Perp_{2} R_{\mathrm{TuC}_{\mathrm{C}}}$

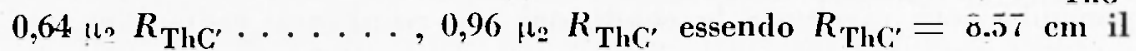
percorso nell'aria delle particelle $\alpha$ emesse dal $T h C^{\prime}$.

Quindi il sistema da risolvere risulta il seguente:

$$
\begin{aligned}
& A_{1} N_{\mathrm{ThC}}+B_{1} N_{\mathrm{RaC}^{\prime}}+C_{1} N_{\mathrm{AcA}}+D_{1} N_{\mathrm{An}}+E_{1} N_{\mathrm{ThA}}+F_{1} N_{\mathrm{AcC}}=n_{1} \\
& A_{2}+B_{2}+C_{2}+I_{2}+F_{2}+C_{3}=n_{2} \\
& A_{3}+B_{3}+C_{3} \\
& A_{4}+B_{4}+C_{4} \\
& A_{5} \quad+P_{5} \\
& A_{6} \quad+B_{6} \\
& \text { A. } \\
& A_{8} \\
& A_{2} \\
& A_{10} \\
& =n_{*} \\
& =n_{4} \\
& =n_{3} \\
& =n_{\mathrm{i}} \\
& =n \text {. } \\
& =n_{\mathrm{s}} \\
& =\boldsymbol{n}_{y} \\
& =n_{10}
\end{aligned}
$$

con i coefficienti $A, B, C, D, E, F$ che si ricavano dalla [2] in hase ai valori di $R$ e di $\mu$; ed essendo $N_{\text {ThC' }} ; N_{\mathrm{Ra} \mathrm{C}^{\prime}}: N_{\mathrm{AcA}}: N_{\mathrm{An}} ; N_{\mathrm{Th} \Lambda}$; $N_{\text {AcC }}$ rispettivamente il numero di particelle a emesse per $\mathrm{cm}^{3} \mathrm{di}$ sostanza in tutto il periodo di esposizione, dal 'Torio $C^{\prime}$, dal Radio $C^{\prime}$; dall'Attinio A, dall'Attinon, dal Torio A e dall'Attinio C.

Il sistema surriportato però $\dot{e}$ indeterminato, pur scmbrando di 10 equazioni e 6 incognite. Limitandolo alle ultime 8 equazioni esso risulta risolvibile nelle tre incognite $N_{\text {ThC: }}, N_{\text {Rat: }}$ e $N_{\text {ACA }}$. Con i valori di queste, e con opportune considerazioni sulle condizioni di equilibrio delle famiglie radioattive, si potreble, con le altre due equazioni, e tenendo conto dei legami intercorrenti fra gli elementi delle famiglie dell'Uranio e dello Attinio, da una parte dedurre i valori di $N_{\mathrm{An}}, \Lambda_{\mathrm{ThA}}$ e $N_{\text {A.r. }}$, e, dall'altra, verificare l'esattezza o meno dell'ammissioni fatte. 
3. -- Si può ancora considerare che il numero di particelle a emesse da ogni elemento della famirlia dell'Attinio è, generalmente, trascurabile rispetto a quello delle particelle $\alpha$ emesse da ogni elemento, contenuto nella stessa roccia, della famiglia dell'Uranio; quindi in pratica può trovarsi $N_{A c A}=N_{A n}=N_{A c C}=u$. in questo caso è possibile risolvere un sistema di 10 equazioni nelle tre incognite $N_{\text {ThC', }} \Lambda_{\text {RaC', }}$ e $N_{\text {ThA }}$ : mentre però per la famiglia del Torio, l'esistenza o meno dell'equilibrio può direttamente dedursi dai valori di $\Lambda_{\text {ThC }}$ e $N_{\text {ThA }}$ (si intende per gli elementi compresi fra il Torio A e il Torio C'), nulla potrà dirsi per la famiglia dell'Uranio.

Dal fatto poi che $N_{\mathrm{ThC}^{\prime}}$ compare in tutte le equazioni, mentre $N_{\mathrm{RaC}}$ vi compare in sei, e $N_{\mathrm{ThA}}$ in due soltanto vi è da aspettarsi, per le tre grandezze, pesi sensibilmente diversi, ed errori medi più forti per $N_{\mathrm{ThA}}$ e $N_{\mathrm{RaC}}$ (in particolar modo per la prima) che non per $N_{\mathrm{ThC}^{\prime}}$.

Con i valori di $n$ ottenuti per lave delle eruzioni vesuviane del 1631, 1855, 1872 e 1944 sono stati ricavati per $N_{\text {AcA }}$ valori non solo compresi nei limiti degli errori, ma anche - com'era da aspettarsi trascurabili rispetto a quelli di $N_{\mathrm{ThC}}$ e $N_{\mathrm{RaC}^{\prime}}$ : il che ha autorizzato a risolvere il sistema nelle tre incornite $\Lambda_{\mathrm{ThC}}, N_{\mathrm{RaC}^{\prime}}$ e $\Lambda_{\mathrm{Th}}$. per quest'ultima grandezza però sono stati dedotti errori medi molto forti, da ragriungere perfino - come nel caso dei campioni delle lave del 1855 e del 1872 - il valore ricavato per l'incognita.

Perciò si sono ritenuti accettabili soltanto $i$ valori calcolati per $N_{\text {ThC' }}$ e $N_{\text {RaC' }}$ che si riportano nella Tabella I.

Tabella I

\begin{tabular}{|c|c|c|c|}
\hline Wittil & $\begin{array}{c}\text { Esposizione } \\
\text { lin seri }\end{array}$ & $N_{\text {The }}$ & ${ }^{\prime} \mathrm{RaC}$ \\
\hline $\begin{array}{l}\text { I631 } \\
1855 \\
1872 \\
1914\end{array}$ & $\begin{array}{l}5,03.10^{6} \\
7,52 \\
5,98 \\
5,03\end{array}$ & $\begin{array}{l}(17,20 \div 0,31) \cdot 10^{5} \\
(25,74 \div 2,42) \\
(17,97 \div 0,76) \\
(43,8 \pm \pm 1,00)\end{array}$ & $\begin{array}{l}(5,7+1,43) \cdot 10^{5} \\
(36,59+11,35) \\
(31,66+3,49) \\
(30,15 \pm 4,78)\end{array}$ \\
\hline
\end{tabular}

Per poler risalire dal valore di $N_{\text {RaC }}$ alla quantita di Radio e rquindi di L'ranio presente, e da Kric alla quantità di Torio, debbono essere fatle alcune ammissioni ( - . Nei casi in esame, oltre a supporre che le famiglie radioattive erano in equilibro nei campioni di lava - prelevati nell'interno di colate delle date indicate - funo all'atto della polverizzazione dei campioni, si può ritenere che alla 


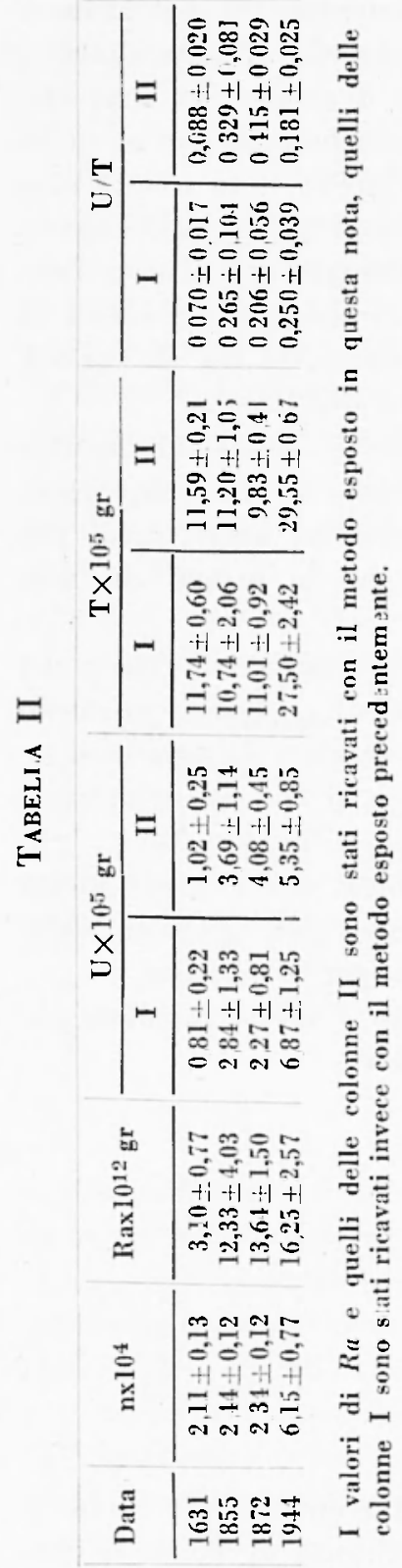

chiusura delle cassettine, contenenti le lastre fotografiche a contatto con le sostanze polverizzate, non erano presenti lo cmanazioni, e che, intine, tutte le quantilà di emanazioni, formantisi nel corso dell'esposizione sono rimaste racchiuse nelle casselline stesse. Percio il numero $\mathrm{N}^{*}$. di atomi di Radio presenti per $\mathrm{cm}^{3}$ è dato da:

$$
N_{\mathbf{R a}}^{*}=\frac{N_{\mathbf{R a C}^{\prime}}}{\left(t-\frac{1}{{ }^{\prime}{ }_{\mathrm{Rn}}}\right){ }^{\lambda} \mathrm{Ra}}
$$

cssendo $t$ il tempo di esposizione $\lambda_{\mathrm{Ra}} \mathrm{e} i_{\mathrm{Rn}}$ rispettivamente le costanti di disintegrazione del Radio e del Radon. Analoghe formule possono ricavarsi per l'Cranio e il Torio, solo che in quest'ultimo caso da tener conto anche del rapporto di ramificazione del Torio C.

Dai valori del peso atomico degrli elementi e della densità della roccia può ottenersi il tenore in Uranio e Torio delle lave in esame; nella Tabclla Il sono riportati insieme ai valori ricavati con il metodo qui descritto, anche quelli ottenuti, con il metodo esposto nella nota precedente, ammettendo l'equilibrio radioattivo nelle lave.

Nella Tabella sono riportati ancora $i$ rapporti in peso $C_{\mathrm{L}} C_{\mathrm{Th}}$ e un indice della radioattivita complessiva data dal numero (n) di particelle a raccolte per $\mathrm{cm}^{2}$ di superficie e per sec aventi la proiczione sulla lastra del percorso nell'emulsione maggiore di $0,60_{\|_{2}} R_{\mathrm{Th}_{1} \mathrm{C}^{\prime}}$.

4. - In conclusione nel far notare come i dati ricavati concordano, nel senso delle variazioni, con quelli trovati dal Joly $\left({ }^{3}\right)$ si mette in evidenza che disponendo di un numero convenientemente elevato di 
tracce - in modo da avere una migliore precisione nelle soluzioni del sistema [3] — è possiloile ricavare direttamente, senza ciò̀ alcuna ammissione "a priori », il numero di atomi di Torio C', Radio C' e Altinio A disintegrati per $\operatorname{cm}^{3}$ di sostanza nel corso dell'esposizione. Con questi valori poi si può calcolare il numero di atomi disintegrati di Attinon, Torio A e Attinio C e contemporaneamente verificare le condizioni dei diversi elementi delle famiglie radioattive rispetto a quelle che sono le condizioni di equilibrio.

In condizioni meno favorevoli riesce possibile dedurre direttamente il numero di atomi disintegrati di Torio C', Torio A e Radio C': è quindi possibile procedere a verifiche soltanto per la famiglia del Torio.

In generale, infine, riesce possibile ottenere valori attendibili del numero di atomi disintegrati di Torio $C^{\prime}$ e Radio $C^{\prime}$ : per risalire al tenore in Uranio e Torio della sostanza si deve necessariamente ricorrere ad ammissioni sulle condizioni di equilibrio di entrambe le famiglie radioattive.

$$
\text { Napoli - Osservatorio Vesuviano - Giugno } 195.3
$$

\section{RIASSUNTO}

In base alle formule date in note precedenti si ricava un sistema di equazioni che permette, dal numero di particelle u raccolte su una lastra fotografica e aventi le proiezioni del percorso nell'emulsione maggiori di detorminate lunghezze, di calcolare il numero di atomi di $\mathrm{ThC}^{\prime}, \mathrm{RaC}^{\prime}$ e AnA disintegrati per $\mathrm{cm}^{3}$ di sostanza in tutlo il tempo dell'esposizione. Si indica come da questi valori si può contemporaneamente dedurre il numero di atomi di An, ThA e AcC disintegrati e verificare le condizioni in cui si trovano $i$ diversi elementi delle famiglie radioattive.

Si mostra poi come con lo stesso sistema in condizioni meno favorevoli è possibile dedurre il numero di atomi disintegrati di ThC', ThA e RaC', e verificare le condizioni della sola famiglia del Torio; ovvero soltanto il numero di atomi disintegrati di $\mathrm{ThC}^{\prime}$ e $\mathrm{RaC}^{\prime}$.

Nel primo caso il tenore in Uranio e Torio può ricavarsi senza fare alcuna ammissione: nel secondo invece bisogna farne soltanto per quel che riguarda la famiglia dell'Uranio; e nell'ultimo, infine, 
necessario farne su entrambe le famiglie radioattive, con l'evidente conseguenza che $i$ valori ricavati assumono significati diversi in ognuno dei tre casi.

\section{SUMMARY}

On the basis of the formulat given in a preceding note, one obtains a system of equations which permits, from the number of alpha particles registered on a photographic plate, and jrom the projections of the paths in the emulsion greater than a certain determined range, the calculation of the number of atoms of $\mathrm{ThC}^{\prime}, \mathrm{RaC}^{\prime}$, and $\mathrm{AnA}$ disintegrating per $\mathrm{cm}^{\circ}$ of the substance during the total exposure time. It is indicated, how, at the same time, one can deduce from these values, the number of atoms of $\mathrm{An}$, ThA, and $\mathrm{AcC}$ disintegrating, and verifv the conditions in which one finds the various elements of the radioactive families.

One then demonstrates hou, with the same system under less favorable conditions; it is possible to deduce the number of disintegrating atoms of $\mathrm{Th}^{\prime}$, $\mathrm{ThA}$ and $\mathrm{RaC}^{\prime}$, and verify the conditions of the single family of Thorium, or else only the number of disintegrating atoms of $\mathrm{ThC}^{\prime}$ and $\mathrm{RaC}^{\prime}$.

In the first case, the content of Uranium and Thorium can be determined without any approximations, in the second, it is necessary to malie approximations only for Uranium family, and in the last approssimations must be made for both radioactive families, with the evident consequence that the values so calculated have " different significance in each of the three cases.

\section{BIBLIOGRAFIA}

(1) Imbò e Casertano, Ann. Geof., vol. III, 475, 1950 e vol. V, 1, 1952.

(2) Nota in corso di stampa su Ann. Oss. Ves, serie VI, vol. I.

(3) Joly, Phil. Mag., 18, 577, 1909. 